\title{
Implications of BRCA Testing in a 27-Year-Old Breast-Feeding Mother With a Strong Family History of Malignancy
}

\author{
J. Drew Payne ${ }^{\mathrm{a}, \mathrm{b}}$, Teri Payne
}

\begin{abstract}
Confidence in identifying and ease of testing for BRCA1 and BRCA2 gene mutations has led to general clinicians ordering BRCA1 and BRCA2 mutations testing more frequently than other cancer genetic tests. When present, these mutations increase breast and ovarian cancer risk dramatically as well as several other cancers. In years past, identification of mutations was saved for an older high-risk patient population; data supporting current management recommendations studied a population mirroring that group. Now, an increasingly younger patient population is having the mutation identified and facing increasingly complicated decisions regarding lifetime risk. Presented here is a case of a 27-year-old breast-feeding mother of two with a strong breast cancer family history, who was found to have an enlarged lymph node and newly identified with a BRCA1 gene mutation. Current recommendation for screening and breastfeeding are presented as well as psychological implication on this increasingly young BRCA1 and BRCA2 gene mutation positive population.
\end{abstract}

Keywords: BRCA1; BRCA2; Breast-feeding; Breast cancer; Ovarian cancer; Implication; Genetic testing; Malignancy

\section{Introduction}

A 27-year-old female presented two months post-partum during an outpatient office visit complaining of pain in her left breast tissue. Her main concern at the time was for infection but she voiced worry about breast malignancy given a strong family history of inflammatory breast cancer. The patient elected to undergo BRCA1 and BRCA2 gene sequenc-

\footnotetext{
Manuscript accepted for publication February 21, 2013

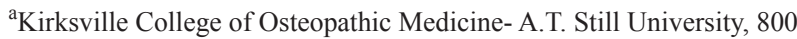
W Jefferson St, Kirksville, MO 63501, USA

${ }^{\mathrm{b}}$ Corresponding author: J. Drew Payne, 7811 Clearmeadow Amarillo TX 79119 Cell- 806-316-3282, USA. Email: japayne@atsu.edu
}

doi: http://dx.doi.org/10.4021/jmc1148w ing which uncovered a deleterious BRCA1 mutation. What psychosocial affect does BRCA1 and BRCA2 testing have on unaffected women? And should unaffected BRCA1 and BRCA2 positive mothers stop breastfeeding to undergo diagnostic screening?

\section{Case Report}

TN, 27, is a mother of two healthy daughters with the most recent labor two months prior. She has been breast-feeding since that time. Both births were induced vaginal deliveries without complication. Prior to presentation, she was without medical concern. She denied drinking or smoking and does not currently take any medications on a regular basis. TN has a strong family history of breast cancer (Fig. 1). Her mother first developed inflammatory breast cancer while pregnant with TN at age 32, 10 years later, at age 42, developed a new primary breast cancer in the previously healthy breast; TN's mother, at age 52, was found to have metastatic lesions from the inflammatory breast cancer. TN has two maternal aunts, both of whom developed breast cancer, one at 57 and the other at 51. BRCA gene mutation testing had been performed on one aunt but information regarding the specific mutation was unavailable. TN works as an $\mathrm{RN}$ and is currently completing her master's degree as a Family Nurse Practitioner. She has been married for five years and resides in a three-bedroom home in the suburbs. No allergies.

Physical examination showed engorged tender left breast with erythematous streaking toward left axillary region. Milky discharge lacking blood or pus was easily expressed from left and right milk ducts. Swollen region on left breast was ropey and warm upon palpation. No palpable masses were found in left or right breast. Right breast exam was unremarkable. No nodules or lymph nodes were appreciated in axillary or supraclavicular regions bilaterally. Remainder of the physical exam demonstrated no pathological findings. Gynecological exam was without masses, tenderness, or abnormalities. No somatic dysfunction was identified on osteopathic exam.

Breast sonography and MRI showed no suggestion of malignancy. An $8 \mathrm{~mm}$ cyst, as well as an enlarged lymph 


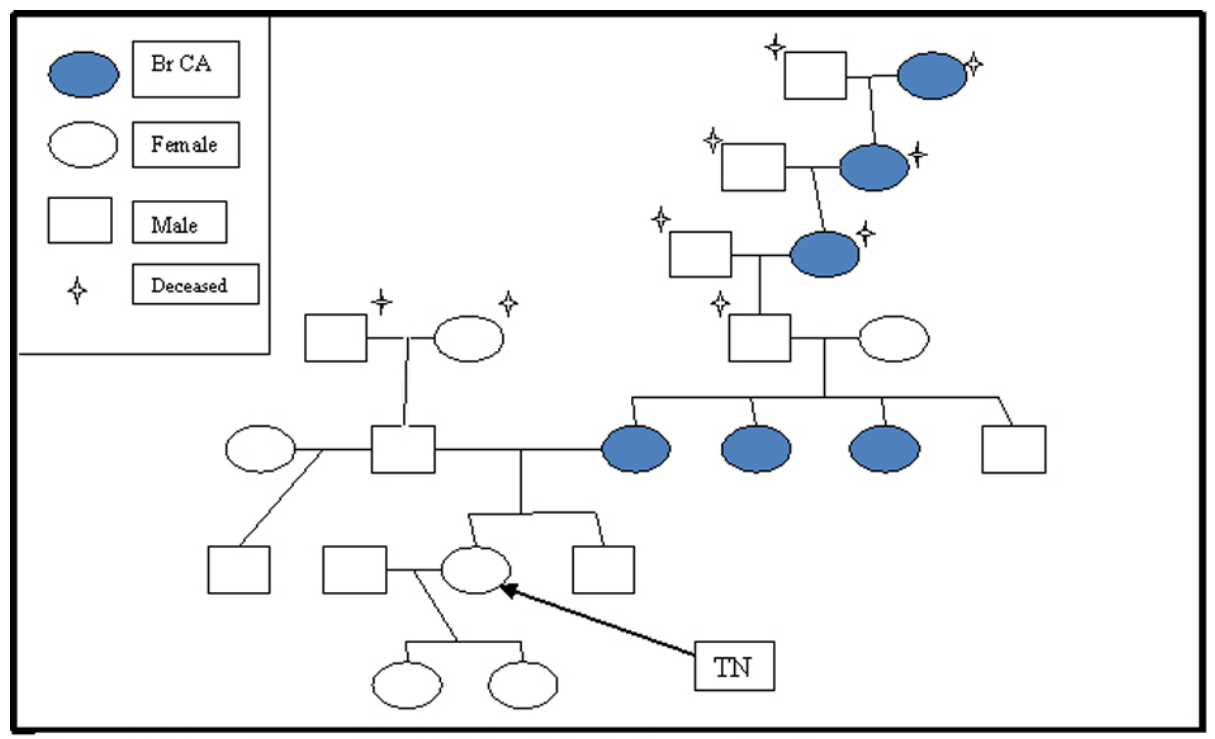

Figure 1. Pedigree of presented patient describing incidence of breast cancer.

node, was appreciated in the upper inner quadrant of the left breast. The lymph node demonstrated cortical thickening suggestive of a reactive lymph node, likely secondary to mastitis. Complete sequencing of BRCA1 and BRCA2 gene was performed as well as testing of five specific genomic rearrangements, not detectable by sequencing. The method revealed a deleterious mutation of a $6-\mathrm{kb}$ insertion involving exon 13, a BRCA-1 mutation.

The patient was given the initial diagnosis of mastitis with further work up needed to rule out malignancy. She was placed on a 14-day regiment of antibiotics and instructed to continue to breastfeed and use a breast pump to empty breasts completely after feeding. Warm moist heat was applied to the breast three to four times a day. In light of the newly found BRCA-1 gene mutation, options regarding alternating six-month screening MRI and mammogram as well as chemo-preventive and prophylactic surgery were discussed with the patient. A consultation with a genetic counselor was requested by the patient. In addition, a consultation with a breast surgeon was requested given the newly found cyst and reactive lymph node. At that time, further information was given regarding prophylactic surgery and risk-benefit of preventative treatments. A conservative attitude was taken given the patient's desire for future children. At the two-week follow up, signs of infection were markedly decreased. The patient had no new complaints regarding the initial symptoms. The patient did, however express concerns regarding quality of screening and safety of screening in regards to concurrent breastfeeding as well as a concern that family members did not understand the implication of the BRCA testing in regards to their own health. Recommendation was made for the patient to continue breast-feeding for as long as she desired.

\section{Discussion}

Breast cancer is the second most common cause of cancerrelated death in American women. In 2011, there were approximately 250,000 new cases of invasive breast cancer and approximately 60,000 cases of in situ breast cancer [1]. In 2011, approximately 40,000 women were expected to die from breast cancer [1]; only lung cancer accounts for more cancer deaths in women. BRCA positive women face an $85 \%$ increase in lifetime breast cancer risk, up from nearly $10 \%$ in the normal population. Risk in ovarian cancer also jumps a dramatic $27 \%$ in a lifetime. In one study, males from BRCA2 positive families have a relative risk 4.7 greater for prostate cancer [2]. The study looked at 173 breast-ovarian cancer families with known BRCA1 and BRCA2 gene mutations finding increases also in pancreatic, gallbladder, stomach carcinoma, as well as melanoma.

\section{Management difficulties}

The management of BRCA1 and BRCA2 gene mutation positive patients begins with two categories of options, surveillance and prevention. Management of testing is especially challenging given the lack of good medical therapy to treat and prevent expression of this mutation. First, highrisk surveillance is recommended in this population. Yearly self-breast exams are recommended at age 18 with clinical breast exams recommended 2 - 4 times annually, beginning at age 25 . Yearly image screening often begins as soon as the mutation is found, no later than age 25. High-risk screening involves annual MRI and mammograms, preferably alternating in six-month intervals. Screening recommendations for ovarian cancer involve twice-yearly transvaginal ultrasound 
and serum CA-125 level at age 35 or earlier, depending on family history of ovarian cancer [3]. Screening by its nature is not perfect. One failure with screening is the inability to detect ovarian cancer early. Transvaginal ultrasound and CA-125 are often recommended but no test or imaging has improved mortality with early detection of ovarian cancer.

Prevention is another component of recommendations. Chemo-prevention with tamoxifen has been shown to lower the chance of developing breast cancer approximately $50 \%$ in women with a moderately increased risk for breast cancer but limited data is available in populations with BRCA1 and BRCA2 gene mutations [4]. Also risk-reducing surgery is recommended in prevention of ovarian and breast cancer. Salpingo-oophorectemy is recommended by age 35 or at the end of child-bearing years. Risk reduction of ovarian cancer has been found to be greater than $90 \%$ [5]. Bilateral total mastectomy is recommended and can reduce risk by $90 \%$. One problem with these recommendations in this case is the young age of the patient. Data are limited in regards to timing of these preventative procedures and the patient faces difficult questions regarding timing of these procedures. Procedures do not come without risks, so patients must be aware of changes to bone and cardiovascular health as well as changes to body image, libido, and surgically induced menopause. There is no crystal ball to indicate when or if a BRCA1 and BRCA2 gene mutation positive woman will develop ovarian or breast cancer, making it difficult to advise when to it is best to have prophylactic surgery.

\section{Family concern}

Unaffected women found to have BRCA1 or BRCA2 gene mutations face challenges regarding not only their own health care, but also the responsibility of informing relatives of their increased risk as well. In our case, the presumed transmission is vertical through the maternal grandfather although we do not have genetic testing to confirm this. Once a novel mutation is identified in a patient, more tailored genetic testing can be offered at a lower cost to siblings who desire to undergo testing themselves. Siblings have a $50 \%$ chance of carrying the same mutation and the same risk of passing the gene onto their offspring. Male-carrier siblings also need to be informed of their risk to pass the gene onto offspring. First cousins are also at increased risk depending on the inheritance pattern of their parents.

\section{Ease to information}

Increasingly, identification of DNA mutations predisposing to cancer is expanding to include whole-genome profiling for personalized approaches to cancer patients and directto-consumer genetic testing [6]. Confidence in identifying BRCA1 and BRCA2 gene mutations has led to general clinicians ordering BCRA1 and BRCA2 mutations testing more frequently [7]. Direct-to-consumer testing places results of testing into the hands of patients often without adequate counseling or legitimate access to reliable information. The newfound ease and access to testing places a large burden on increasingly younger patients. Young patients, as in this case, must consider when or if to have preventative surgeries and must take into account future child planning. Large life decisions and understanding of what these mutations mean for long-term health is essential. Young women with no detectable disease are faced with a choice of bilateral mastectomy and salpingo-oopherectomy as a means of reducing risk, not curing disease. In one case, a 29-year-old female with no identifiable disease had elected for risk reducing bilateral total mastectomy and hysterectomy with adnexectomy [8]. Medical personnel must be familiar with specialized testing in order to properly counsel patients. Often variations exist in the personal interpretation of a result, leading to various clinical recommendations, and suggest a need for clinical management recommendations as well [9]. The U.S. Preventive Services Task Force (USPSTF) and American College of Medical Genetics has outlined broad guidelines on appropriate testing and follow up but recommendations are limited for younger age groups, as in our patient's case [10]. It is clear the information is available but needs adjunctive counseling and support through medical personnel [6].

\section{Psychological}

Rarely brought to the attention of the provider is the effect of genetic testing to family dynamic and psycho-social environment. To "label" a patient as increased risk for a cancer is difficult news to give and receive. It uncovers questions that can never be full answered; "When will I get cancer?" and "Will I pass the gene on to my children?" Often patients eligible for testing are all too aware of their positive family history and consider being diagnosed with cancer as a "when" not "if" situation. Then, to label them as increased risk because they inherited a gene from a family member, compounds the issue. Although unintended, family members can feel guilt or worry that they have or someday will pass the gene on. The saying "ignorance is bliss" does not ring untrue for some patients in regards to genetic testing. Yet, as health providers, knowledge is often our only weapon against risk and future disease. We must be prepared for implications of any test we order.

\section{Breast-feeding}

A special problem in this case was the patient's concern that lactating breast tissue will distort imaging and "hide" an occult cancer. The long-term benefit of breast-feeding has been shown in multiple studies. One analysis included individual data from 47 epidemiologic studies including 50,302 women with invasive breast cancer and 96,973 controls found an es- 
timated relative risk reduction of breast cancer by $4.3 \%$ for every 12 months of breast-feeding, as well as a decrease of $7 \%$ for each birth [11]. Benefits of breast-feeding have also been shown in osteoporosis and cardiovascular disease as well as the economic benefit to parents. Benefits to the infant immune system and gastrointestinal tract are also well supported with current data. In this healthy patient, screening was recommended according to the same guidelines with the understanding that images might be less predictive. Specific concerns of change in breast tissue that does not fluctuate with hormone cycles will need to be investigated on a case-by-case basis. Patients should be encouraged to take action in life style actions to reduce their risk of future breast and ovarian cancer. Actions such as these will not only reduce risk, but also empower patients to take charge of their health.

\section{Summary}

BRCA1 and BRCA2 gene mutation testing and other genetic testing will open the door to better understanding of diseases that have long been battled by health-care providers and patients. Our job as providers is to partner with patients as an advocate and educator. Opportunities exist to empower BRCA positive patients with screening and preventative measures but it is important for providers to understand not only the pathophysiology of testing, but also the psychology of it.

\section{References}

1. American Cancer Society. (2012). Breast cancer facts \& figures 2011-2012. Atlanta, GA: American Cancer Society, Inc.

2. Cancer risks in BRCA2 mutation carriers. The Breast Cancer Linkage Consortium. J Natl Cancer Inst. 1999;91(15):1310-1316.

3. Daly MB, Axilbund JE, Buys S, Crawford B, Farrell CD, Friedman S, Garber JE, et al. Genetic/familial highrisk assessment: breast and ovarian. J Natl Compr Canc
Netw. 2010;8(5):562-594.

4. Isaacs, C., Fletcher, S., \& Peshkin, B. (2012). Management of hereditary breast and ovarian cancer s y n drome and patients with BRCA mutations. UpToDate: Wolters Kluwer Health. Retrieved from http://www.uptodate.com/contents/management-of-hereditary-breastand-ovarian-cancer-syndrome-and-patients-with-brcamutations? source=search_result\&search=Management + of + hereditary + breast + and + ovarian + cancer $+\% 09$ syndr ome + and + patients + with + BRCA + mutations\&selectedTi tle $=1 \sim 150$

5. Rebbeck TR, Lynch HT, Neuhausen SL, Narod SA, Van't Veer L, Garber JE, Evans G, et al. Prophylactic oophorectomy in carriers of BRCA1 or BRCA2 mutations. N Engl J Med. 2002;346(21):1616-1622.

6. Surbone A. Social and ethical implications of BRCA testing. Ann Oncol. 2011;22(Suppl 1):i60-66.

7. Cox SL, Zlot AI, Silvey K, Elliott D, Horn T, Johnson A, Leman RF. Patterns of cancer genetic testing: a randomized survey of Oregon clinicians. J Cancer Epidemiol. 2012;2012:294730.

8. Zubor P, Kajo K, Dussan CA, Szunyogh N, Galo S, Danko J. Repetitive demand for radical cancer risk reduction surgery in a young BRCA1 mutation carrier with strong family history of BRCA linked malignancies. Ginekol Pol. 2006;77(7):543-549.

9. Petrucelli N, Lazebnik N, Huelsman KM, Lazebnik RS. Clinical interpretation and recommendations for patients with a variant of uncertain significance in BRCA1 or BRCA2: a survey of genetic counseling practice. Genet Test. 2002;6(2):107-113.

10. American College of Medical Genetics Foundation. (1999). Genetic susceptibility to breast and ovarian cancer: Assessment, counseling and testing guidelines. Retrieved from http:/www.health.ny.gov/diseases/cancer/ obcancer/contents.htm.

11. Breast cancer and breastfeeding: collaborative reanalysis of individual data from 47 epidemiological studies in 30 countries, including 50302 women with breast cancer and 96973 women without the disease. Lancet. 2002;360(9328):187-195. 